\title{
Change of strain rate and seismicity in the Chubu district, central Japan, associated with a Tokai slow event
}

\author{
Tomokazu Kobayashi* and Manabu Hashimoto \\ Disaster Prevention Research Institute, Kyoto University, Gokasho, Uji, Kyoto 611-0011, Japan \\ (Received June 14, 2006; Revised February 14, 2007; Accepted February 27, 2007; Online published June 8, 2007)
}

\begin{abstract}
We have evaluated temporal variation in strain rates in the Chubu and Kinki districts, central Japan, between 1997 and 2004 using continuous GPS data of GEONET. We found that strain rates throughout the southern part of Chubu district, including the Yoro fault and Nobi fault zone, have significantly deviated from the secular crustal deformation pattern since at least 2001. The results of an inversion procedure show that the strain rate change can be well explained by a slow slip beneath the Tokai area, suggesting that the Tokai slow event has significantly affected the crustal deformation not only in the Tokai area but also in an area located more than $100 \mathrm{~km}$ from the main slip area of the event. We also found that the seismicity rate has clearly decreased by approximately $50 \%$ around the Yoro fault since the latter half of 2000. The $\triangle$ CFF inferred from our Tokai slow slip model agrees with the observed reduction in seismicity. These results were interpreted as indicating that the seismicity, which has been decreasing for several years, is controlled by the reduction in the shear stressing-rate due to the Tokai slow slip.
\end{abstract}

Key words: Strain rate change, seismicity change, Tokai slow event, static stress change.

\section{Introduction}

Since the second half of 2000, continuous Global Positioning System (GPS) observations have detected anomalous crustal deformations in the southern coastal area of the Tokai region, located in the southern part of Chubu district, central Japan about $200 \mathrm{~km}$ southwest of Tokyo (Fig. 1(a)). It has been inferred that the varying rate of surface motion is mainly caused by a slow slip event that occurs on the plate boundary where the Philippine Sea plate is subducting under the Eurasian plate beneath the Tokai region (Ozawa et al., 2002). The total released moment is estimated to be equivalent to a moment magnitude of 7, as of 2005 (Ozawa et al., 2005). It is possible that such a large slip has some effect on a surrounding stress field. Recent studies have shown that a static stress change due to a large earthquake correlates well with seismic activity - that is, the seismicity rate increases where the stress increases and falls off where the stress drops-as in the 1992 Landers, California earthquake (Harris and Simpson, 1992; Jaume and Sykes, 1992; Stein et al., 1992), the 1994 Northridge, California earthquake (Stein et al., 1994), and the 1995 Kobe earthquake (Hashimoto, 1997; Toda et al., 1998). This suggests that seismic activity can be sensitive to a stress change. In actual fact, the seismicity did change in the Tokai region in and around the subducting plate during the anomalous slow

\footnotetext{
*Now at Institute of Seismology and Volcanology, Graduate School of Science, Hokkaido University, Kita 10 nishi 8, Kita-ku, Sapporo, Hokkaido 060-0810, Japan.

Copyright (c) The Society of Geomagnetism and Earth, Planetary and Space Sciences (SGEPSS); The Seismological Society of Japan; The Volcanological Society of Japan; The Geodetic Society of Japan; The Japanese Society for Planetary Sciences; TERRAPUB.
}

event (Matsumura, 2002, 2005).

However, the inland crustal deformation in the Chubu and Kinki districts within a few hundred kilometers from the slip area has not been studied in any detail. In terms of the released moment, a significant crustal deformation is possibly induced by the slow event in not only the area surrounding the slip but also in areas distant from the slip. Additionally, there are several inland faults in the Chubu and Kinki districts (Fig. 1(b)). In particular, microearthquakes are relatively active around the Yoro fault and the Nobi fault zone, both located about $200 \mathrm{~km}$ from the slip area (Fig. 1(b)). If a change in the inland strain field and/or the seismicity is related to the slow event, it can provide a good example for assessing the relationship between inland earthquakes and thrust events along the trench.

In this study, we have therefore investigated how the Tokai slow event has affected the surrounding area, in addition to the Tokai region itself. With this aim, we have evaluated the temporal variations of strain field and seismicity, and constructed the corresponding slow slip model, even though several researchers have already modeled the slow slip event, so as to quantitatively estimate the relation between the aseismic event and the strain/seismicity change. We initially estimated the strain rate in the Chubu and Kinki districts using GPS data, and examined its temporal variations. We then obtained a slow slip distribution beneath the Tokai region by conducting an inversion in order to derive the relationship between the estimated strain rate change and the Tokai slow event. With these data, we were able to investigate the seismicity change and, finally, to discuss the relationship between the seismicity change and the static stress changes caused by the slow event. 
(a)

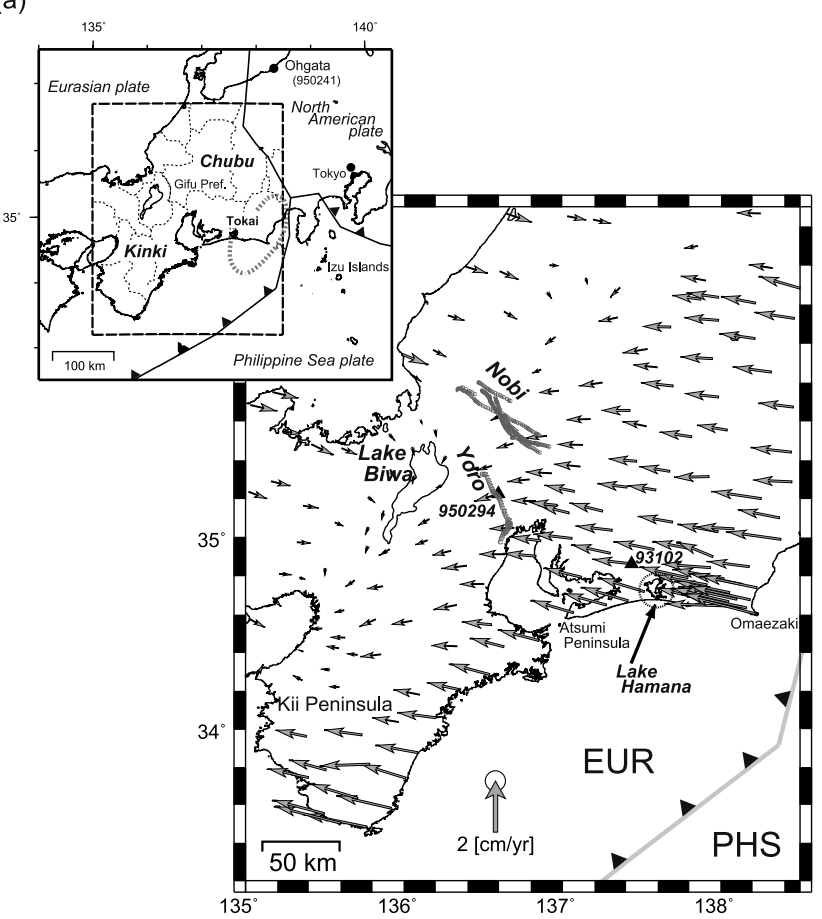

(b)

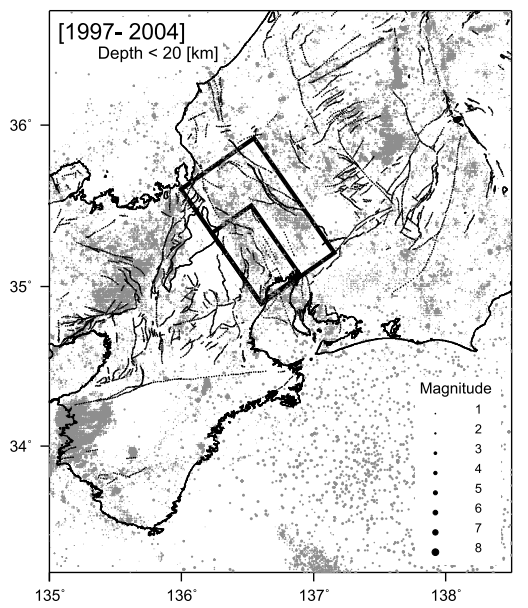

Fig. 1. (a) (Left top) Location of the Chubu and Kinki districts and tectonic setting in and around the Japanese islands. A rectangle indicates the study area. The anticipated source area of the Tokai earthquake is indicated by the hatchured line. (Right bottom) Gray arrows represent displacement rates of each GPS station from January, 1997 to May, 2000 with reference to the Ohgata GEONET station (950241). Error ellipses show the 95\% confidence levels. The Yoro and the Nobi fault zones are indicated by thick lines. (b) Seismicity map and inland faults in Chubu and Kinki districts. Earthquakes shallower than $20 \mathrm{~km}$ from 1997 to 2004 are shown.

\section{Strain Rate Distribution}

\subsection{Data and method}

In order to estimate the crustal deformation in the Chubu and Kinki districts, we used F2 solutions of GEONET (GPS Earth Observation Network) which has been deployed and functional throughout the Japanese islands by the Geographical Survey Institute (GSI), Japan. We extracted the data from stations located $134-139^{\circ}$ longitude and $33-37^{\circ}$ latitude so that the analyzed domain covers the Chubu and Kinki districts. We initially estimated the averaged horizon- (a)

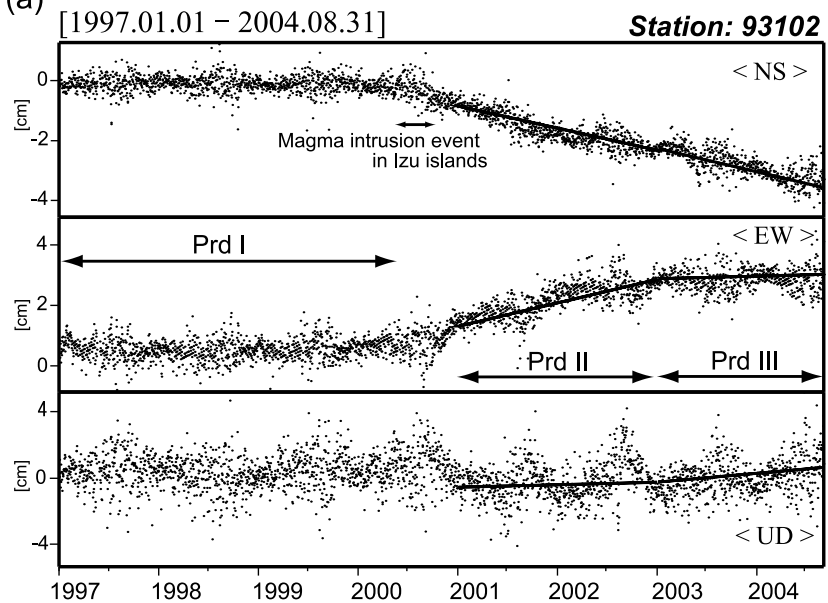

(b)

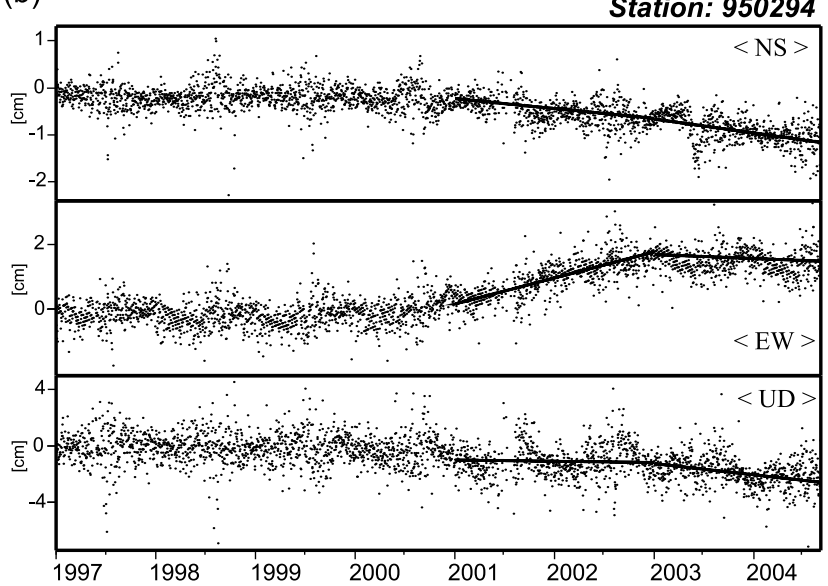

Fig. 2. Examples of time series data of daily coordinates of GPS sites from January 1997 to August 2004. Three-component data at the sites 93102 and 950294 indicated by solid triangles in Fig. 1(a), are shown. The linear trend and annual variation components estimated from January 1997 to May 2000 are removed from the time series data. The solid lines in Prds II (January 2001 to December 2002) and III (January 2003 to August 2004) indicate the averaged trends derived from a linear fitting.

tal displacement rates by describing the time series data as a combination of a linear trend, a sinusoidal seasonal component, and coseismic steps. In this study, any corrections of steps associated with earthquakes were not executed because earthquakes that produced significant ground surface deformations did not occur during the period of our study. However, there are some steps in the GPS time series data caused by the replacement of antennas, so we modeled the steps due to the artifacts as coseismic steps. In this manner, we obtained horizontal displacement rates for each component with a least square method. Figure 1(a) shows the estimated displacement rates at each GPS station, with reference to Ohgata (950241), from January 1997 to May 2000 as an example of the obtained displacement rates

We next evaluated strain rates by applying the method of Shen et al. (1996) in which the spatial smoothing procedure is used. Using this approach, we solved an observation equation that an observed velocity at an observation point is related to the horizontal displacement rate, strain rates, and a rotation rate at an arbitrary point on a two-dimensional plane. Here, the observational errors are weighted by a 

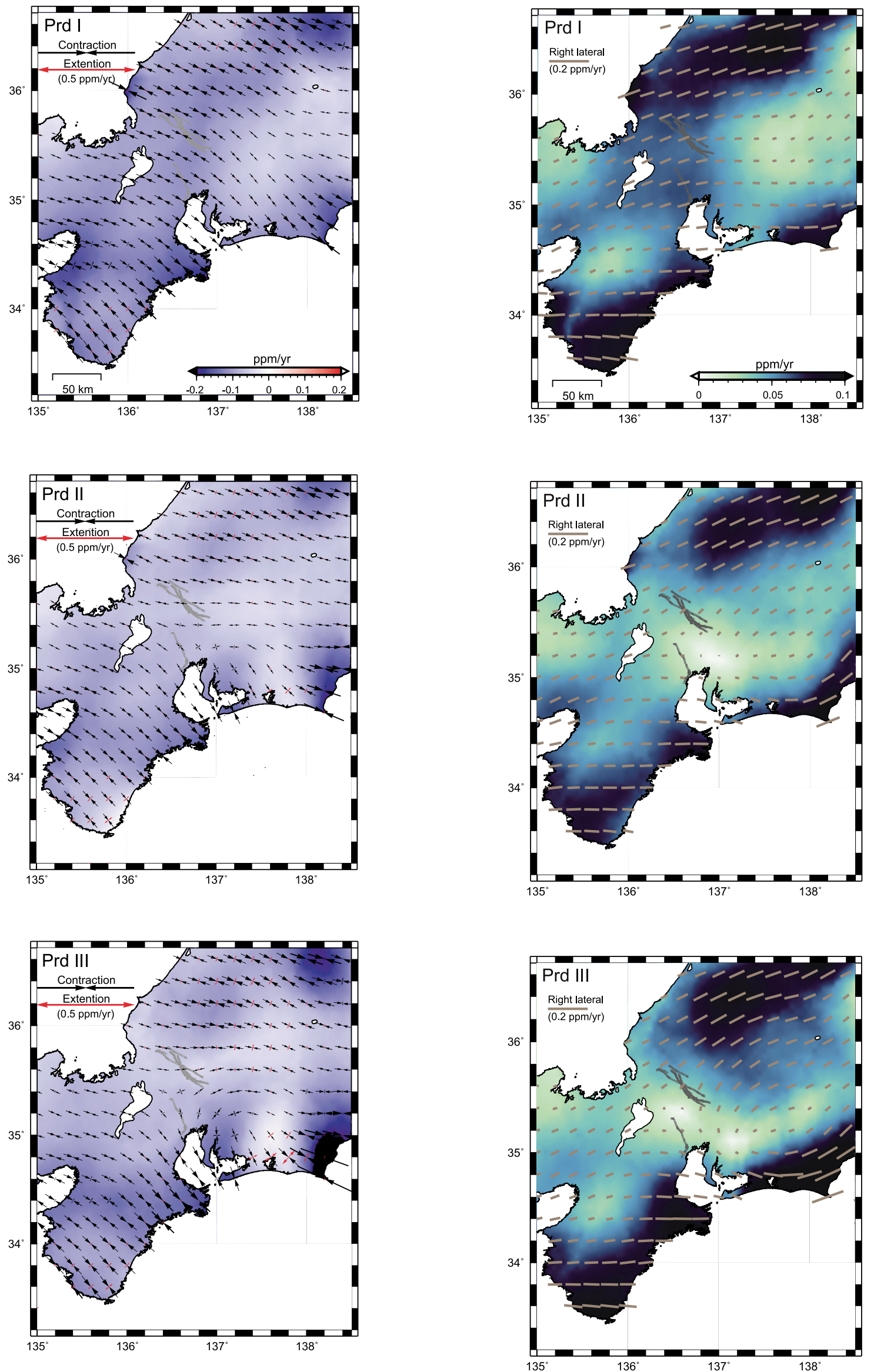

Fig. 3. Maps showing the distribution of dilatation and principal axes of strain rates for Prds I, II, and III, respectively. Colder colors denote smaller values of the dilatation rate. Black and red arrows represent contractional and extensional strain, respectively. The color scale is the same for all three maps.

Fig. 4. Maps showing the distribution of maximum shear strain rate for each period of time. Lighter colors denote smaller values. Solid lines represent the direction and magnitude of the right lateral component. 
(a)
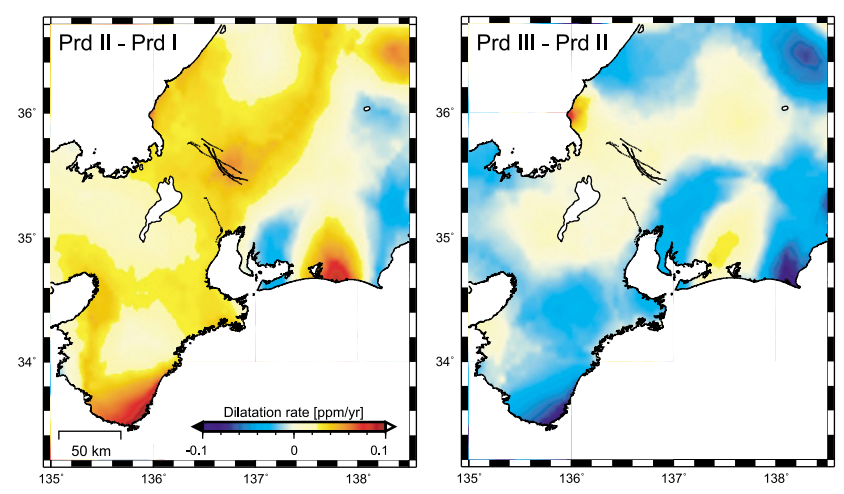

(b)

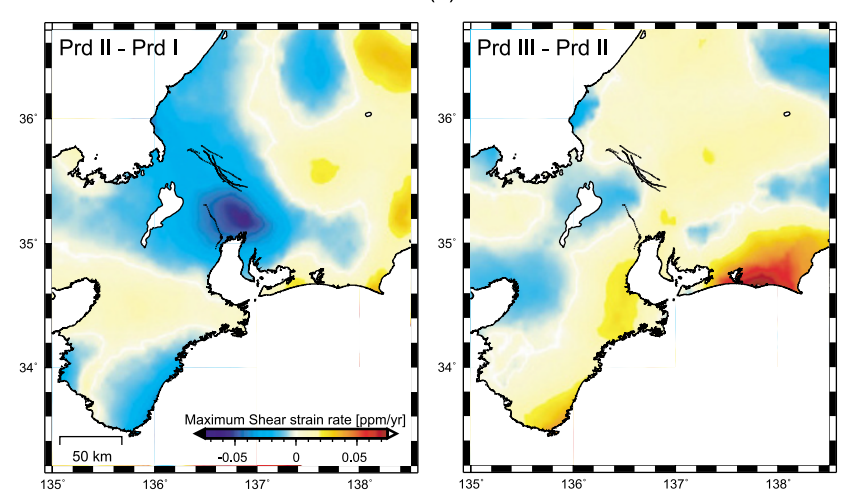

Fig. 5. (a) The difference in the dilatation rate between two periods of time. Left panel, the strain rate of Prd I is subtracted from that of Prd II; right panel, as in the left panel, but for Prd II from III. (b) Same as Fig. 5(a) but for the maximum shear strain rate.

Gaussian distribution depending on the distance $\Delta R$ between a station and a calculation point and are defined as $\sigma_{x, y} \exp \left(\Delta R^{2} / 2 D^{2}\right)$ (Sagiya et al., 2000), where $\sigma_{x, y}$ is the original observational error of the $x$ or $y$ component of the displacement rate, and $D$ is a smoothing distance parameter that controls the weight among the observations to give a larger weight to a closer station. We applied $30 \mathrm{~km}$ as $D$. Stations within a distance of $2 D$ from a calculation point are used to estimate the strain rate components.

Figure 2 shows the time series of daily coordinates at GPS stations 93102 and 950294. The former is located near Lake Hamana, and the latter is $100 \mathrm{~km}$ distant from the lake (Fig. 1(a)). The linear trend and annual variation components estimated from January 1997 to May 2000 are removed from the time series data. Thus, values close to zero mean that the GPS site moves with the secular velocity, while non-zero values mean motions that are different from the secular rate. This figure shows that the values from 1997 to the first half of 2000 are close to zero; conversely, from the latter half of 2000 onwards, the inclination significantly deviates from 0 . Furthermore, the motion is not at a constant rate. The inclination at both stations in the EW component changes once again in 2003. We therefore set three periods of time-January 1997 to May 2000 (Prd I), January 2001 to December 2002 (Prd II), and January 2003 to August 2004 (Prd III) — and then estimated the averaged strain rates for each period. We did not use the period from
June to December in 2000 and after September 2004 in order to avoid the effects of the dyke intrusion event in the Izu volcanic islands (e.g. Nishimura et al., 2001; Ozawa et al., 2004) and the two large earthquakes that occurred southeast off the Kii peninsula, Japan, on 5 September 2004 (Mw = 7.3 and 7.5; Hashimoto et al., 2005), respectively. Our purpose was to compare the temporal variation of strain rates in each period, thus we used common GPS stations over the study period from Prd I to III for this estimation in order to avoid the effects of different station configurations. We consequently removed the stations deployed after 1999 and anomalous sites and ultimately used data obtained from 256 GPS stations for the estimation.

\subsection{Strain rate distribution in the Chubu and Kinki} districts

Figure 3 shows the estimated dilatation and principal strain rate, and Fig. 4 shows the maximum shear strain rate. The features of the estimated strain rates are summarized as follows.

2.2.1 Dilatation rate: Figures 3 and 5(a) show the dilatation rate for each period of time and the difference of the rate between two periods, respectively. The strain rate of Prd I is subtracted from that of Prd II in the left panel of Fig. 5(a); similarly, the strain rate of Prd II is subtracted from that of Prd III in the right panel. The color code shows the magnitude of the dilatation rate, with colder colors representing smaller values. The dilatation rates have a negative sign in Prd I throughout the whole region, indicating that a contractional deformation is dominant. The rate, however, increases from Prd I to II by several tens of percentage points, indicating that the contractional deformation is weakened. We can first identify that the area of weakened contraction is around the Nobi fault zone. During Prd III, the contractional deformation also remains weakened in the region, although the amount of change is relatively smaller. For example, the dilatation rates for the Nobi fault zone are estimated as $-10.5 \pm 0.8 \times 10^{-2}$, $-6.4 \pm 0.9 \times 10^{-2}$, and $-5.4 \pm 1.2 \times 10^{-2} \mathrm{ppm} /$ year for Prds I, II, and III, respectively. For the Yoro fault, the rates are $-11.0 \pm 0.7 \times 10^{-2},-7.7 \pm 0.8 \times 10^{-2}$, and $-7.6 \pm 1.2 \times 10^{-2}$ $\mathrm{ppm} /$ year for Prds I, II, and III, respectively. We use two points for determining these rates, $\left(136.6^{\circ} \mathrm{E}, 35.6^{\circ} \mathrm{N}\right)$ and $\left(136.6^{\circ} \mathrm{E}, 35.2^{\circ} \mathrm{N}\right)$, which represent the Nobi fault zone and the Yoro fault, respectively. Figure 6(a) shows the error of the dilatation change; the top and bottom panels represent the error in the change from Prd I to II and from Prd II to III, respectively. One $(1 \sigma)$ and two $(2 \sigma)$ standard deviations are calculated for each period. The errors in the area where the strain rate changes shown in Fig. 5(a) do not exceed $1 \sigma$ or $2 \sigma$ are not plotted; that is, the rate change in the colored area is significant at each confidence level. Warmer colors indicate smaller errors. From Prd I to II, the rate changes around the Nobi fault and the Yoro fault zone are rather reliable because they exceed $2 \sigma$. On the other hand, from Prd II to III, the dilatation changes around the two fault zones are approximately comparable to $1 \sigma$, so that the rate change would not necessarily be significant. In addition to the area around the two fault zones, we can find large dilatation changes in the Tokai region and the southern tip of the Kii Peninsula. In the Tokai region, the contrac- 
(a)
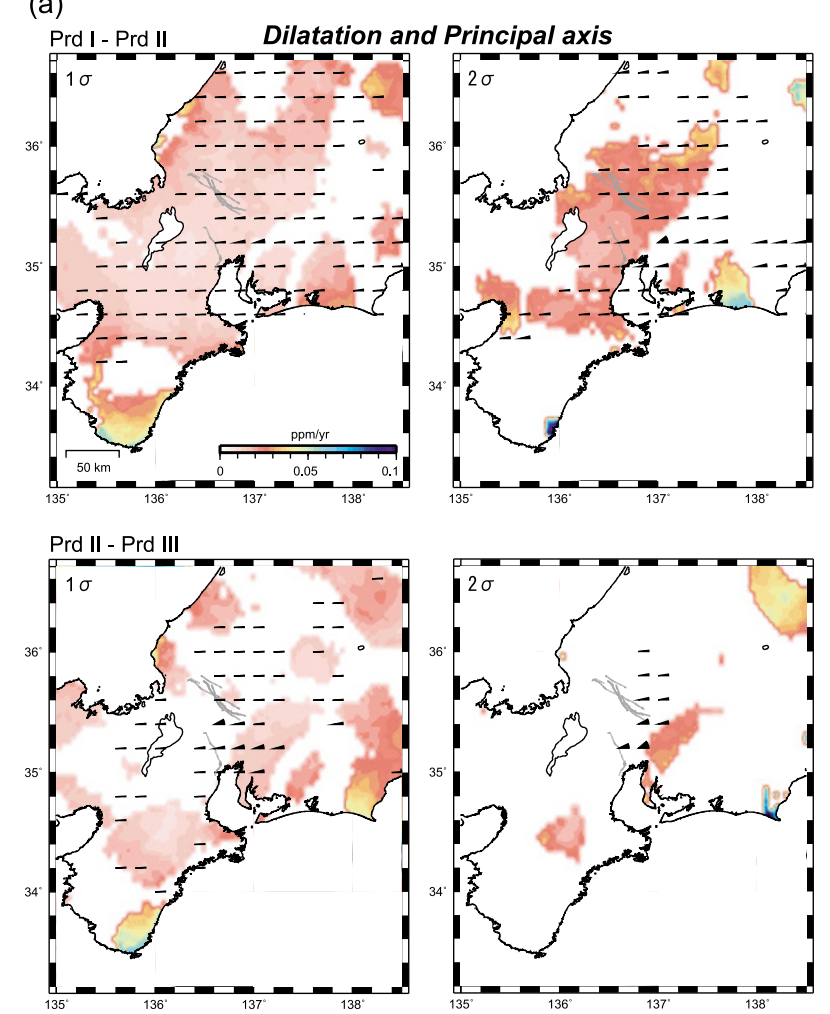

(b)

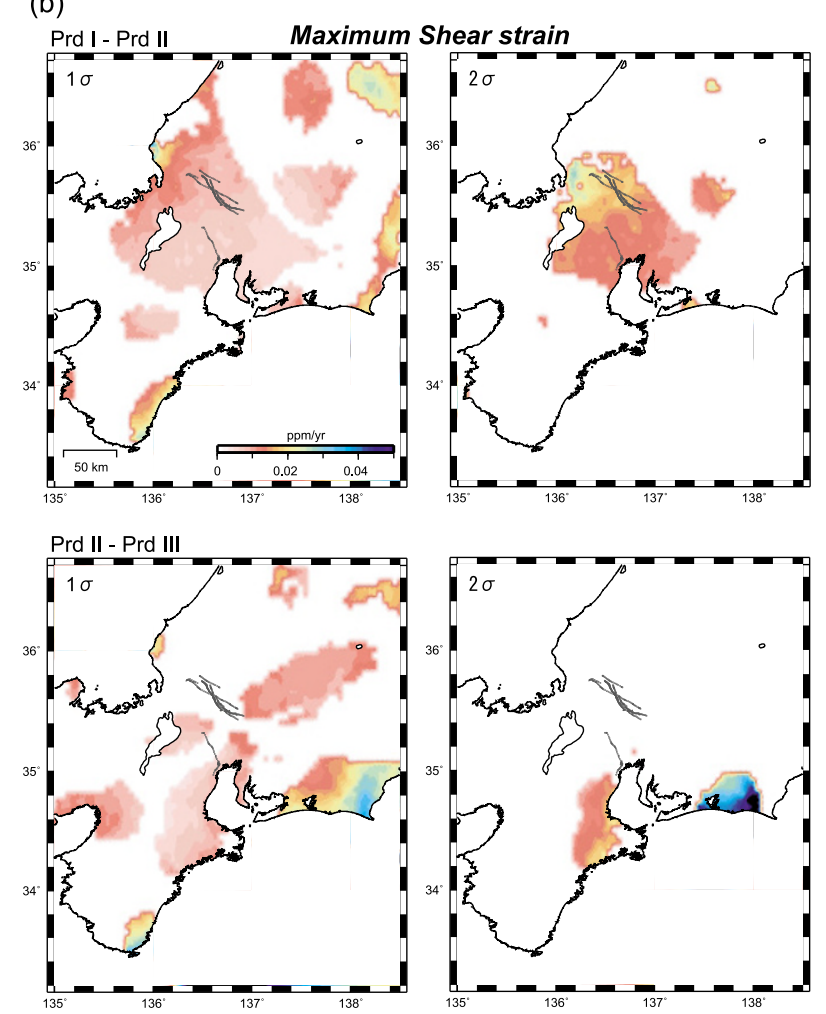

Fig. 6. (a) Maps showing the distribution of the error in the dilatation rate change from Prd I to II (top) and from Prd II to III (bottom). Colder colors represent larger values. The $1 \sigma$ error and $2 \sigma$ error are shown on the left and right, respectively. Black fans denote the angle error of the principal axes of strains. The errors in the area where the strain rate/angle changes do not exceed $1 \sigma$ or $2 \sigma$ are not plotted. (b) Same as in Fig. 6(a) but for the errors of the maximum shear strain change.

tional deformation around Lake Hamana decreases. In Prd III, the expansion rate around Lake Hamana becomes larger, and around the Omaezaki, the contractional deformation increases. The characteristics of strain change in the Tokai region are consistent with the results of Yoshikawa (2003). The change in the Kii Peninsula reaches $0.1 \mathrm{ppm} / \mathrm{year}$, but does not exceed $2 \sigma$.

2.2.2 Principal strain rate: The estimated principal strain rate axes are also shown in Fig. 3 for each period. Black and red arrows indicate contraction and extension, respectively. In Prd I, the contractional component is dominant, on an order of $0.1 \mathrm{ppm} / \mathrm{year}$, and the directions are generally NW-SE to WNW-ESE in the whole Chubu and Kinki districts, reflecting the tectonic loading. From Prd I to II, however, the contractional component decreases by several tens of percentage points in the Chubu district. The decrease is quite remarkable around the Nobi fault zone. In addition, the directions of the axes change from NW-SE to EW. In Prd III, this change in the directions of the axes proceeds further around the Yoro fault, and the extensional component becomes dominant at some points. For the Yoro fault, the azimuths of the maximum principal contractional axes are estimated to be $120 \pm 2,134 \pm 7$, and $168 \pm 11^{\circ}$ for Prds I, II, and III, respectively; for the Nobi fault zone, these are $115 \pm 2$ to $96 \pm 3$ and $85 \pm 5^{\circ}$, for Prds I, II, and III, respectively. The black fans in Fig. 6(a) denote the angle error of the principal axes. The significant change around the two fault zones from Prd I to II can now be identified; it is also possible to identify the significant angle change ex- ceeding $2 \sigma$ around the Yoro fault and the southern region of the Nobi fault zone from Prd II to III. Consequently, we can determine that the contractional axis changes its direction from WNW-ESE to N-S around the Yoro fault and to ENE-WSW around the Nobi fault zone. On the other hand, in the Tokai region, there are no significant changes in the directions of the principal axes, although the extension is quite distinct. In Prd III, the extension becomes larger, and the contraction rate increases around Omaezaki.

2.2.3 Maximum shear strain rate: Figure 4 shows the distribution of the maximum shear strain rate. The difference in the rate between two periods of time is plotted in Fig. 5(b). The color code shows the magnitude of the rate, with lighter colors representing smaller values. The solid lines represent the directions of the right-lateral shear. The estimated maximum shear strain rates also significantly decrease in the Chubu district from Prd II onwards. The pattern of the rate change, however, is slightly different from the dilatation rate - that is, the decrease is dominant around the Yoro fault, with estimated rates of $6.2 \pm 0.7 \times 10^{-2}$, $1.8 \pm 0.8 \times 10^{-2}$, and $1.8 \pm 1.2 \times 10^{-2} \mathrm{ppm} /$ year for Prds I, II, and III, respectively; for the area around the Nobi fault zone, these are $5.8 \pm 0.8 \times 10^{-2}, 3.5 \pm 0.7 \times 10^{-2}$, and $3.4 \pm 1.2 \times 10^{-2} \mathrm{ppm} / \mathrm{year}$ for Prds I, II, and III, respectively. The maximum shear strain rate falls to approximately one third around the Yoro fault. As shown in Fig. 6(b), the decrease in the shear strain around the two faults is rather reliable from Prd I to II. 


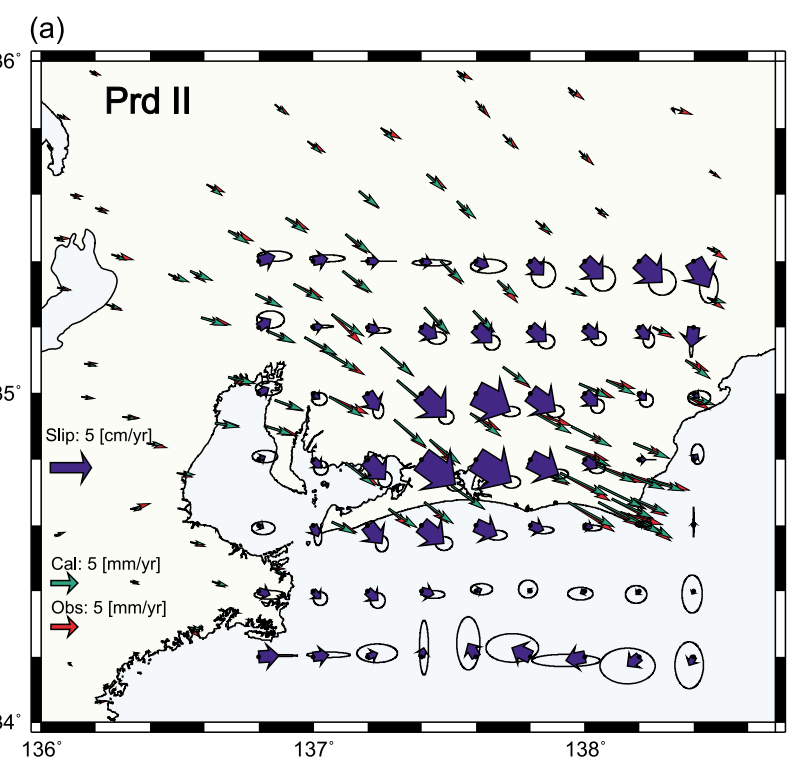

(b)

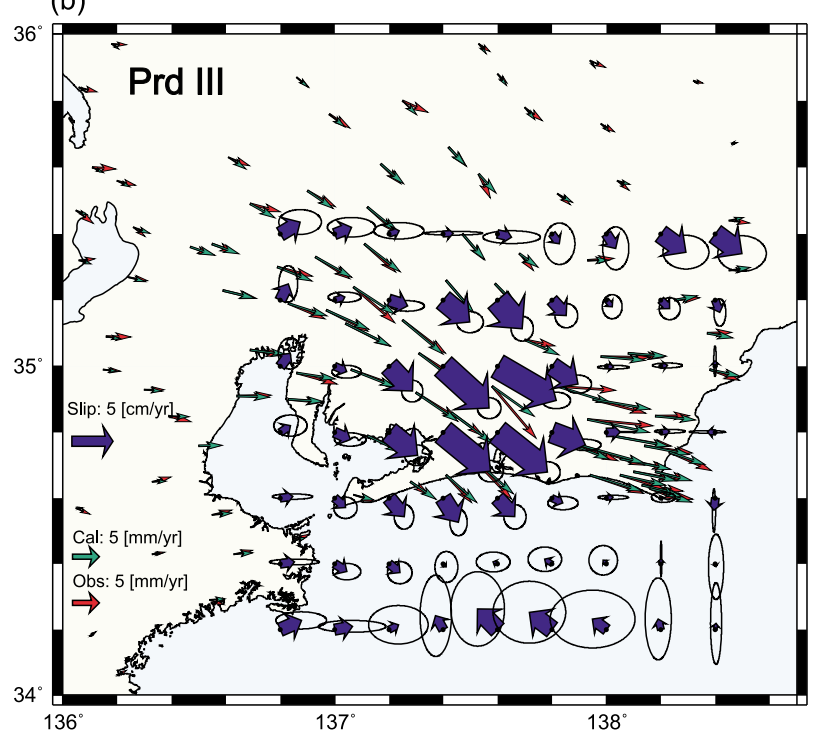

Fig. 7. (a) Slip distribution of the Tokai slow slip event. Thick blue arrows represent the magnitudes and directions of slip rate for each grid point on the plate boundary. Error ellipses on the vectors show the 95\% confidence levels. Red and green arrows are the observed and predicted displacement rates, respectively.

\section{Relationship Between Strain Rate Changes and the Tokai Slow Event}

\subsection{Inversion method for the Tokai slow event}

We found that the strain rates in the Chubu district significantly deviate from the secular crustal deformation pattern. The temporal variations in the strain rate seem to have a strong relationship with the Tokai slow event, always taking into consideration that the event started around 2001 and has continued since then. With this knowledge, we next examined the cause of the strain rate change in order to determine whether or not the strain rate change can be explained by the Tokai slow event. For the analysis, we assumed that the slow event occurred on the plate boundary interface between the Eurasian and Philippine Sea plates below the Tokai region and conducted the following Bayesian inversion procedure. Using the non-secular horizontal dis- placement rates for Prds II and III, we determined the spatial distribution of slow slip. In this calculation, the motion for each period is the deviation from the secular displacement rate in Prd I. We set point sources on the boundary with an interval of $0.2^{\circ}$ from $136.8^{\circ}$ to $138.4^{\circ}$ longitude and from $34.2^{\circ}$ to $35.4^{\circ}$ latitude. We fixed the strikes and dips of each source in advance. For the configuration of the plate boundary interface, we referred to Ishida (1992). Under this condition, we estimated the magnitudes and rakes of slips for each source by a least square method. The increase in model parameters may give rise to instability of the solution, so we imposed a smoothness constraint on the slip distribution to stabilize the solution. We used the following objective function, minimized in the inversion,

$$
\mathbf{O}=(\mathbf{d}-\mathbf{G m})^{\mathrm{T}} \mathbf{E}^{-1}(\mathbf{d}-\mathbf{G m})+K^{2} \mathbf{m}^{\mathrm{T}} \mathbf{W} \mathbf{m},
$$

where $\mathbf{m}, \mathbf{d}$ and $\mathbf{G}$ are the unknown slip vector, observation data vector, and the corresponding Green's function matrix based on Okada (1985), respectively. We assumed that the observed data were mutually independent and that the errors obeyed a Gaussian distribution, $N\left(0, \alpha^{2} \mathbf{E}\right)$, where $\alpha^{2}$ is an unknown scale factor for the variance $\mathbf{E}$. W is a smoothing matrix consisting of a Laplacian operator that smoothes the roughness of the fault slip distribution, and $K$ is the relative weight of the constraints. To determine the value of $K$ objectively, we adopted the minimum Akaike's Bayesian information criterion (ABIC) (Akaike, 1980). For our problem, the ABIC value is defined by

$\mathrm{ABIC}=N \log \mathbf{O}(K)-M \log K^{2}+\log \left|\mathbf{G}^{\mathrm{T}} \mathbf{E}^{-1} \mathbf{G}+K^{2} \mathbf{W}\right|+C$,

where $N$ and $M$ are the number of data and model parameters, respectively. $C$ is a constant term independent of $K$. The search for the value of $K$ that minimizes the ABIC is carried out numerically by an iterative process because we cannot obtain the optimal $K$ to minimize the ABIC analytically. For the observation data, we used 173 GPS stations located in the range $136.0-138.5^{\circ}$ longitude and $34.0-36.0^{\circ}$ latitude, covering the area where the strain rate significantly decreases.

\subsection{Slip distribution and predicted strain rate}

The results of the slip distributions are shown in Fig. 7. Thick blue arrows denote the calculated slip rates on the plate boundary interface. The error ellipses on the vectors indicate the 95\% confidence level, and the small red and green arrows are the observed and predicted non-secular horizontal displacement rates, respectively. The calculated displacement rates are consistent with the observed rates for each period. During Prd II, the main slip area is located beneath Lake Hamana where the maximum slip rate reaches as much as $5.6 \mathrm{~cm} / \mathrm{year}$. For Prd III, the main slip area is still located beneath the lake, but the slip area has been slightly extended to deeper levels. The shift in the slip area was remarked on in earlier studies of the Tokai slow event (Ozawa et al., 2002; Yoshikawa, 2003). The slip rate is increasing, and the maximum value is estimated as 8.8 $\mathrm{cm} /$ year. The released moment rates are estimated to be 2.1 and $2.5 \times 10^{19} \mathrm{Nm} /$ year (equivalent to $\mathrm{Mw}=6.8$ and 6.9) for each period of time, respectively, when assuming a rigidity of $40 \mathrm{GPa}$. The total cumulative released moment is estimated to be equivalent to $\mathrm{Mw}=7.2$. This estimated 
(a)
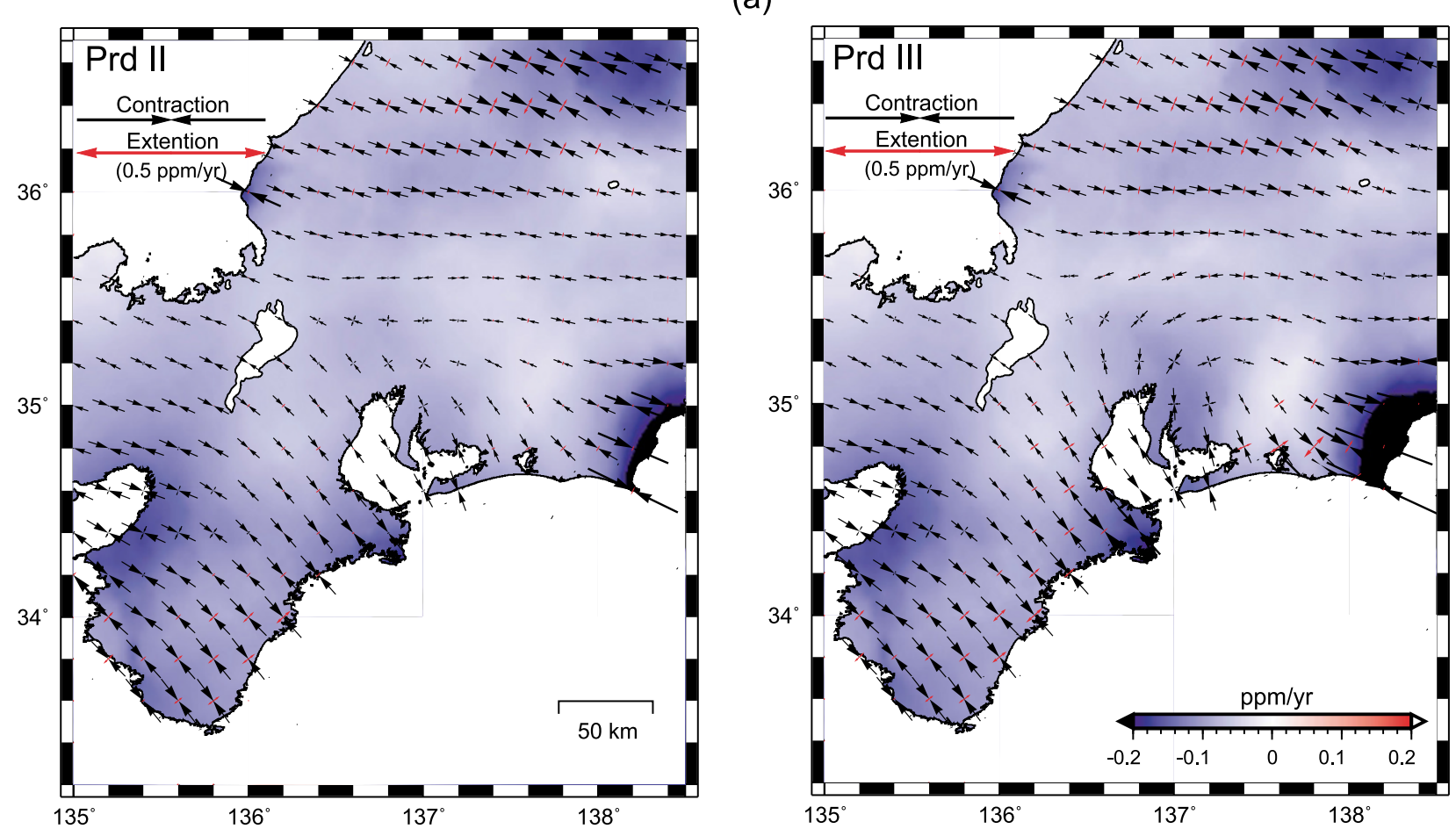

(b)
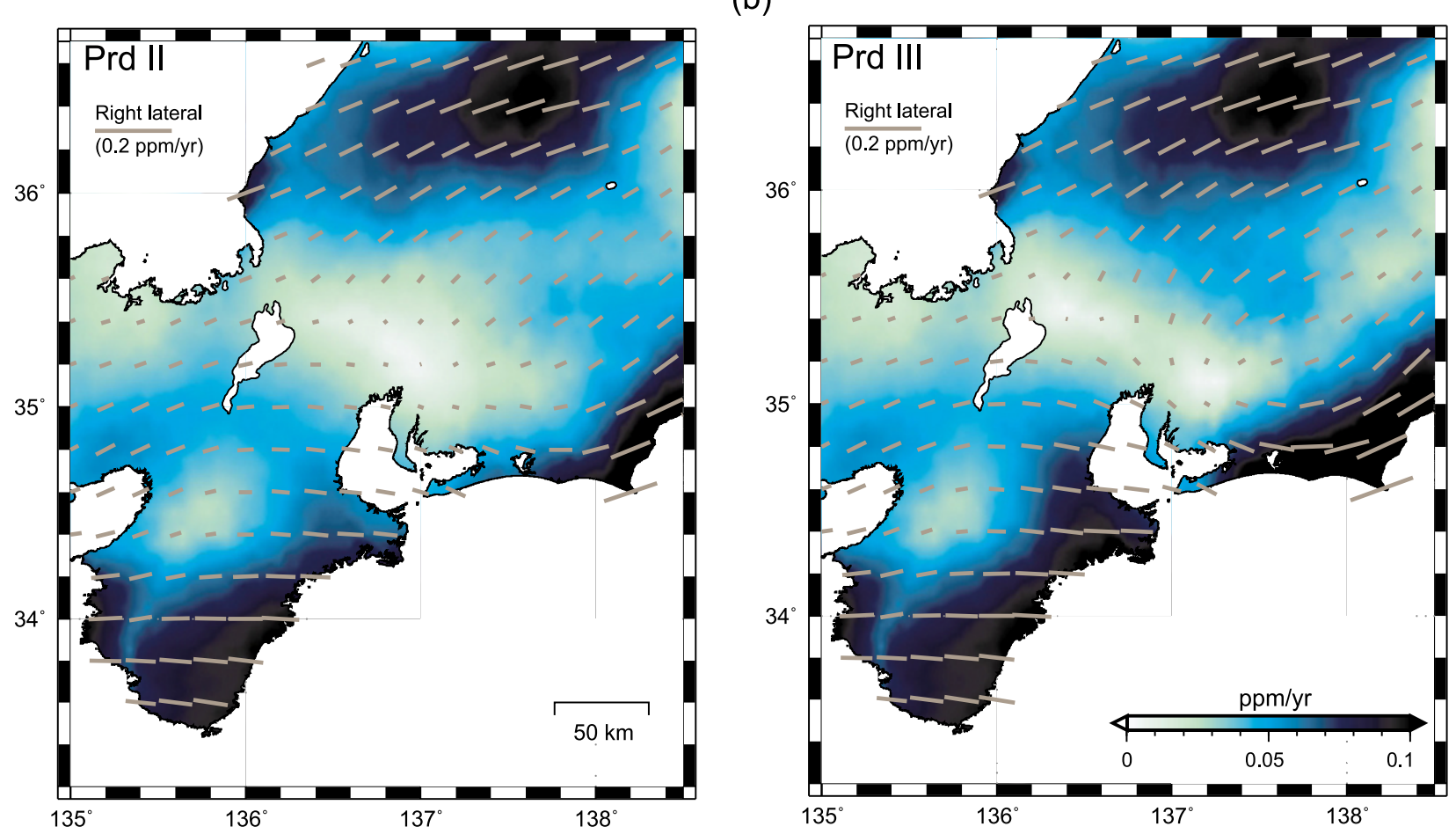

Fig. 8. (a) Maps showing the distribution of predicted dilatation and principal strain rates derived from the slip model shown in Fig. 7. (b) Maps showing the distribution of predicted maximum shear strain rate. The color scales are the same as Figs. 3 and 4.

value is reasonably consistent with the results of Ozawa $e t$ al. (2005) $(\mathrm{Mw} \approx 7.2)$.

The predicted strain rates derived from our inversion results are shown in Fig. 8. We added each predicted displacement rate to each observation in Prd I and obtained the predicted strain rate in the same manner as described in Section 2. The estimated slip on the plate boundary can reproduce well the change in the observed strain rates. For example, the dilatation rates and its azimuths for the Yoro fault are estimated to be $-8.1 \times 10^{-2} \mathrm{ppm} / \mathrm{year}$ and $142^{\circ}$, and $-7.9 \times 10^{-2} \mathrm{ppm} / \mathrm{year}$ and $168^{\circ}$ for Prds II and III, respectively; for the Nobi fault zone, these are $-6.4 \times 10^{-2}$ $\mathrm{ppm} / \mathrm{year}$ and $89^{\circ}$, and $-5.0 \times 10^{-2} \mathrm{ppm} / \mathrm{year}$ and $72^{\circ}$ for Prds II and III, respectively. 
(a)

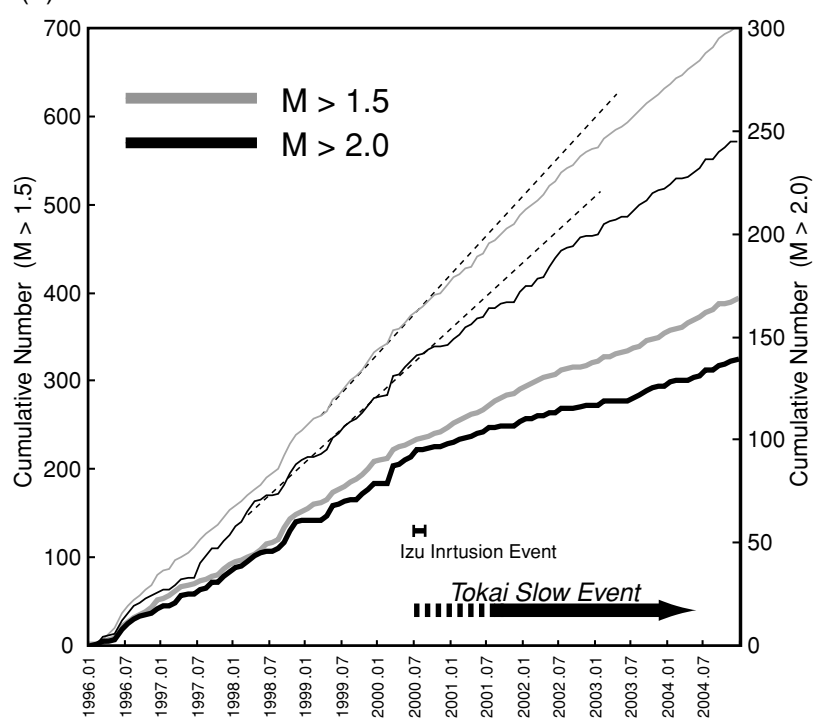

(b)

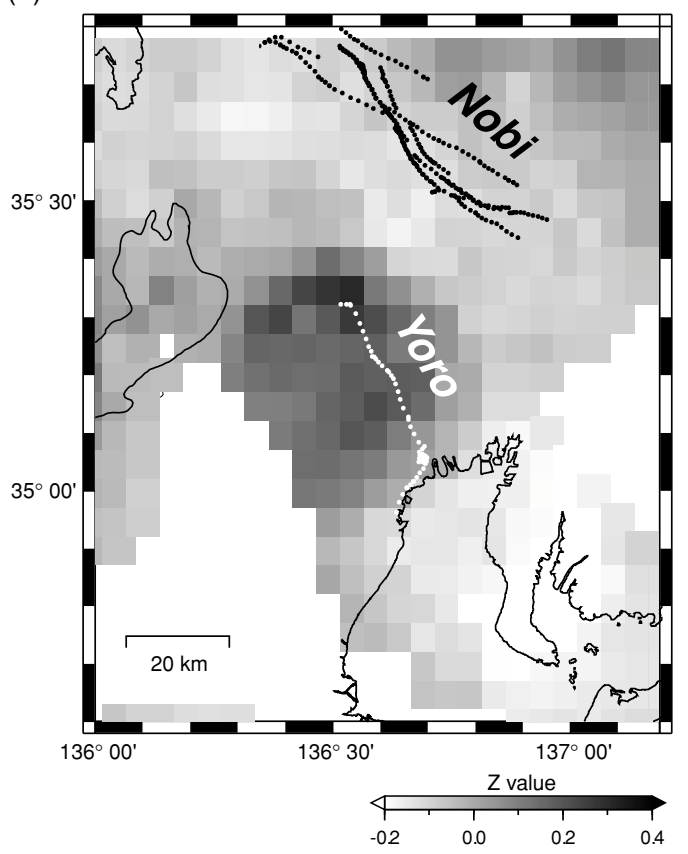

Fig. 9. (a) Cumulative numbers of earthquakes around the Yoro fault and the Nobi fault zone from January 1996 to August 2004. Solid black and gray thin lines represent the cumulative numbers of $\mathrm{M}>2.0$ and $M>1.5$ events, respectively, within the large rectangle in Fig. 1(b). Thick lines show the cumulative numbers within the small rectangle in Fig. 1(b). Left and right vertical axes indicate the cumulative numbers of $\mathrm{M}>1.5$ and $\mathrm{M}>2.0$ events, respectively. Several parameters used for the declustering procedure are as follows: $\tau=10$ days, $P=0.95$, and $Q=10$. The notations follow those by Reasenberg (1985). (b) $z$-value distribution for $M>1.5$ earthquakes around the Yoro fault and Nobi fault zone. Darker colors represent larger values. The two periods of time compared are January 1996 to May 2000 and January 2001 to August 2004.

\section{Seismicity Change}

\subsection{Seismicity change around the Yoro and Nobi faults}

The inversion result suggests that the slow event has a significant effect on the crustal deformation, not only in the Tokai area, but also in regions more than $100 \mathrm{~km}$ from the slow slip area. In order to determine whether the Tokai slow event induces any stress changes that cause significant changes in the seismicity rate, we investigated the seismicity in the area where the strain rate shows significant change. The searched domain is indicated by a larger rectangle shown in Fig. 1(b), where the Yoro fault and the Nobi fault zone are located and the microearthquakes are relatively active. Using earthquake data from 1996 obtained by the Japan Meteorological Agency (JMA), we extracted earthquakes shallower than $20 \mathrm{~km}$ in depth. Since the depth of the plate boundary beneath the region is 30-50 km (Miyoshi and Ishibashi, 2004), the selected earthquakes occur in the crust. To reduce transient seismic activity, including aftershocks and earthquake swarms, we used the declustering procedure of Reasenberg (1985) for the original earthquake catalog and then evaluated the temporal variations of the background seisimicity. The cumulative numbers of earthquakes after the declustering procedure are shown in Fig. 9(a). The black and gray thin lines represent the cumulative numbers of earthquakes larger than $\mathrm{M}=2.0$ and $\mathrm{M}=1.5$ (JMA magnitude), respectively. For $\mathrm{M}>2.0$, a clear decrease is identified from the latter half of 2000. The occurrence rates estimated by a line fit are 2.60 $\pm 0.02 /$ month (January 1996 to May, 2000) and $2.03 \pm 0.02 /$ month (January 2001 to August 2004). Here, the transition period of the decrease is removed, i.e. June 2000 to December 2000. The corresponding decrease is also identified for $\mathrm{M}>1.5$.

To test the spatial distribution of the occurrence of the seismic quiescence statistically, we show the distribution of $z$-values for the $M>1.5$ earthquakes around the Yoro fault and the Nobi fault zone (Habermann, 1983). The $z$-value stands for the statistical significance of the rate change between two periods of time. When the seismicity decreases or increases, the $z$-value has positive or negative values, respectively. We applied the method to the declustered earthquake catalog, and calculated the $z$-values at each grid point with an interval of $0.05^{\circ}$. Earthquakes within a distance of $25 \mathrm{~km}$ from each grid point were used for the estimation, but grid points with less than 50 events were removed. The spatial distribution of the $z$-values is shown in Fig. 9(b). Darker colors represent larger values. The $z$-value is larger near the Yoro fault than in any other area, while it is near zero around the Nobi faults. The statistical results show that the degree of seismicity reduction around the Yoro fault is quite distinct and that there is no significant change in seismicity around the Nobi fault zone. The earthquakes in the region surrounding the Yoro fault, indicated by a smaller rectangle in Fig. 1(b), are extracted, and those cumulative numbers are plotted by thick lines in Fig. 9(a). The abrupt decrease in the seismicity can be clearly identified from the middle of 2000 . The occurrence rates for $M>2.0$ and $M>$ 1.5 change from $1.70 \pm 0.02$ to $0.78 \pm 0.01 /$ month and from $4.39 \pm 0.05$ to $2.85 \pm 0.03 /$ month, respectively; as such, they show a 46 and $65 \%$ decrease, respectively.

Such a change in the seismicity dating from the middle of 2000 has also been detected in the Tokai region (Matsumura, 2002, 2005). Given the large stress change in this region, the cause of the seismicity change is most likely the Tokai slow event. However, an important characteristic of the seismicity change is that this decrease can be de- 

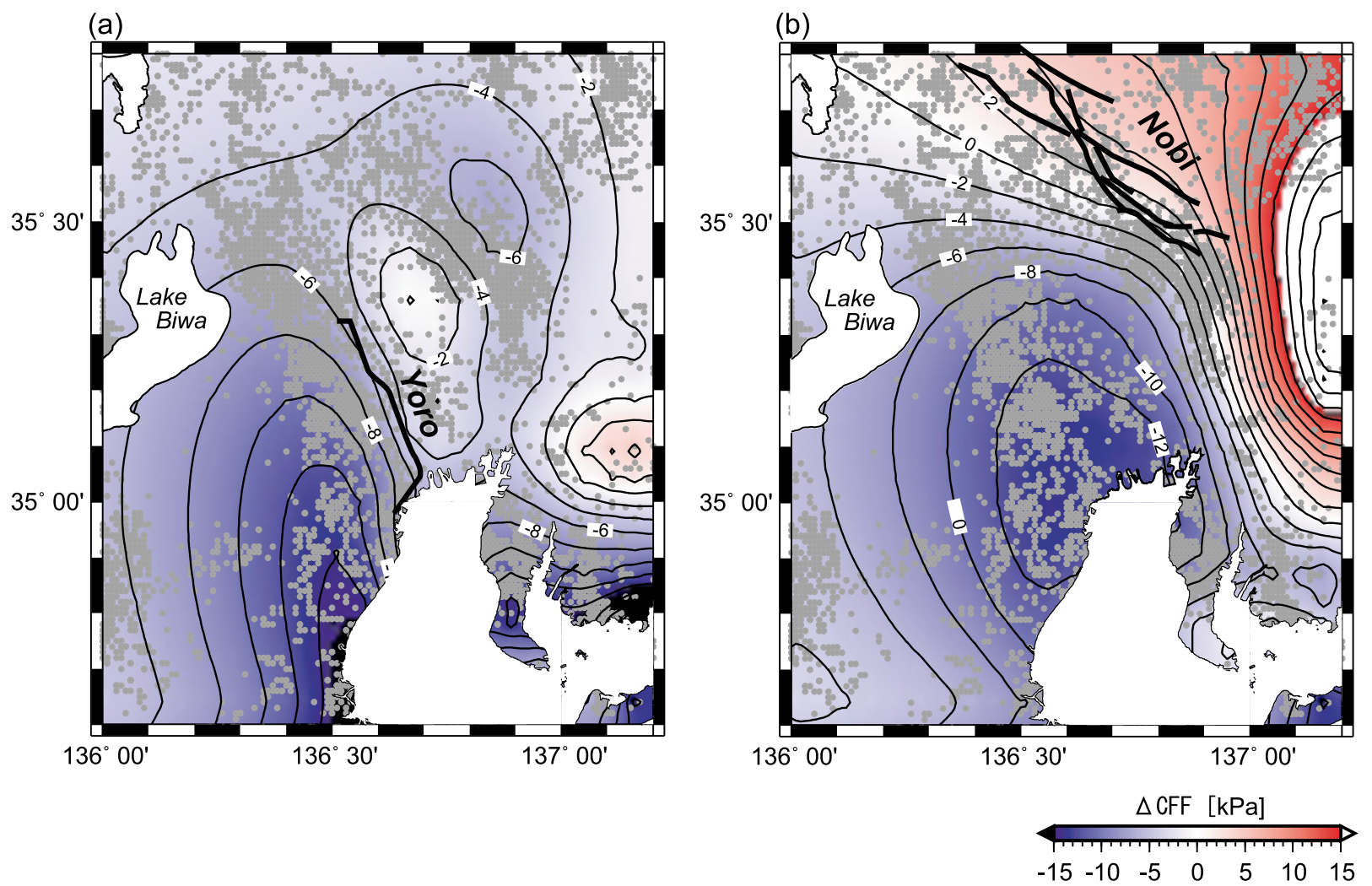

Fig. 10. (a) Spatial distribution of $\triangle \mathrm{CFF}$ for the Yoro fault, derived from the slip model in Fig. 7. Colder colors represent smaller values. The units of the stress values are $\mathrm{kPa}$. The assumed fault geometry for the estimation is $150^{\circ}$ as strike, $45^{\circ}$ as dip, and $90^{\circ}$ as rake. The effective coefficient of friction $\mu^{\prime}$ and rigidity are assumed to be 0.4 and $40 \mathrm{GPa}$, respectively. Small dots represent the earthquakes shallower than $20 \mathrm{~km}$. (b) Same as in Fig. 10(a) but for the Nobi fault zone. The assumed fault geometry for the estimation is $120^{\circ}$ as strike, $90^{\circ}$ as dip, and $0^{\circ}$ as rake.

tected not only in the Tokai region but also around the Yoro fault, which is about $150 \mathrm{~km}$ from the main slip area. Therefore, to investigate the relationship between the seismicity change and the static stress changes caused by the Tokai slow event more quantitatively, we evaluated a Coulomb Failure Function change $(\triangle \mathrm{CFF})$.

\subsection{Static stress change due to the Tokai slow event}

The $\triangle \mathrm{CFF}$ can be used as an indicator for assessing whether the stress change is conducive to slip on an assumed fault plane. The value is defined as $\triangle C F F=$ $\Delta \tau-\mu^{\prime} \Delta \sigma_{n}$, where $\Delta \tau, \Delta \sigma_{n}$ and $\mu^{\prime}$ are the change in shear stress for the slip on the fault plane in the slip direction, the change in normal stress, and the effective coefficient of friction including pore pressure effect, respectively. Here, the normal stress has a positive sign in the case of compression. We assumed 0.4 as $\mu^{\prime}$, according to Stein et al. (1994), and obtained the stress in a homogeneous half space based on the strain derived from our slip model for the Tokai slow event, assuming a rigidity of $40 \mathrm{GPa}$. We then evaluated $\triangle \mathrm{CFF}$ for an assumed fault plane. The source mechanisms of microearthquakes around the Yoro fault and the Nobi fault zone are not well known. Referring to the geometries of the fault, we thus assumed the strike, dip, and rake as $150^{\circ}, 45^{\circ}$, and $90^{\circ}$, and $120^{\circ}, 90^{\circ}$, and $0^{\circ}$, for the Yoro fault and Nobi fault zone, respectively. Here, the strike is measured clockwise from the north. The $\triangle \mathrm{CFF}$ is estimated at the depth of $10 \mathrm{~km}$, applying the total slip for Prds II and III. Figure 10 (a) and (b) show the $\triangle \mathrm{CFF}$ for the Yoro fault and Nobi fault zone, respectively. Colder colors represent smaller values. The $\triangle \mathrm{CFF}$ has a negative sign around the Yoro fault, with a value of -10 to $-5 \mathrm{kPa}$, while the $\Delta \mathrm{CFF}$ for the Nobi fault zone is near zero. Since the region around the Yoro fault is completely in the stress-shadow, the spatial distribution of the $\Delta \mathrm{CFF}$ caused by the Tokai slow slip event agrees with the reduction of the seismicity around the Yoro fault.

\section{Discussion}

The static stress change deduced from our Tokai slow slip model explains well the reduction in seismic activity, but many studies have shown that the $\triangle \mathrm{CFF}$ values of more than $10 \mathrm{kPa}$ can affect seismicity (e.g., Stein, 1999; Toda and Stein, 2002). However, the estimated $\triangle \mathrm{CFF}$ values for the Yoro fault are able to reach final values of only -10 $\mathrm{kPa}$ in a period of 4 years. If the seismicity rate change depends on the magnitude of the stress change itself, the seismic activity must respond to a much smaller stress than the suggested critical value, even though uncertainties may remain in the assumed fault geometry and the slip distribution model. The scenario of the seismicity rate may rely on a rate-state friction law. According to the formulation of Dieterich (1994), a stress transfer model incorporating the rate-state friction suggests that the seismicity rate is dependent on the shear stressing-rate acting on the fault plane, in which the rate change is linearly related to the stressingrate. In the physical process, the seismic activity can vary with the change of the stressing-rate (Toda et al., 2002), even if the CFF change does not reach the above critical 
value generally thought to affect seismicity rates. Our interpretation is that the reduction in the stressing-rate due to the Tokai slow slip has caused the background seismicity rate to keep decreasing for several years. The seismicity rate change from Prd II to III is not as clear as that from Prd I to II, perhaps because the stressing-rate change is much smaller for the former time interval than for the latter. The seismicity rate change had already begun by the middle of 2000 , in association with the non-stable movement that has almost the same trend as the one after 2001 that we have analyzed here (Fig. 2). This suggests that a similar change in stressing rate had also occurred in this period so that the seismicity could be suppressed. The non-stable movement in the middle of 2000 is thought to be contaminated by both the slow slip beneath the Tokai region and the Izu intrusion event (Kobayashi et al., 2005), and the intrusion event also gives a stress shadow for the Yoro fault when the $\triangle \mathrm{CFF}$ is evaluated on the basis of the dyke geometry estimated by Nishimura et al. (2001). It is difficult to distinguish these two effects from existing data because of their resemblance in the deformation patterns. The seismicity change in the middle of 2000 would be influenced by both the events.

In terms of the geodetic studies for the Tokai region, it is possible that at least two similar slip events occurred during the periods 1980-1982 and 1988-1990, based on the analyses of the past baseline measurements by electromagnetic distance meters and the tide gauge data (Kimata et al., 2001; Kobayashi and Yoshida, 2004). Matsumura (2005) revealed that the seismicity around Lake Hamana was also inactivated for the individual periods of the past slip events. We can not recognize the temporal variation in the seismicity from the JMA catalog because the locations of microearthqukes at that time are not determined well due to the poor coverage of seismometers, but a reduction in seismic activity around the Yoro fault may have taken place in association with the stress change. Furthermore, similar changes in the seismicity would be expected to accompany the impending Tokai earthquake for which a $\mathrm{M}=8$ class earthquake is expected. The source area of the large earthquake is thought to be adjacent to the Tokai slow slip area (Fig. 1(a)), and the slip direction is almost the same as that of the slow event. When we set a simple rectangular fault plane based on the anticipated source area and roughly evaluate the strain change, assuming a slip equivalent to a $\mathrm{M}=8$ earthquake, the $\triangle \mathrm{CFF}$ in this region is estimated to be $-100 \mathrm{kPa}$. Thus, it is likely that a significant decrease in the seismicity would be induced in the area.

The ability to monitor the inland seismicity before and after the Tokai slow event and the impending Tokai earthquake will play an important role in the future for gaining a further understanding of the essential relationship between the seismicity and the stress change being applied to the source region, and between inland earthquakes and thrust events along the trench.

\section{Conclusions}

We have evaluated the strain rates and seismicity in the Chubu and Kinki districts and investigated its temporal variations. The following conclusions were derived from the analyses:
1) The contractional deformation has been significantly weakened from at least 2001 in and around the southern Chubu district and the Tokai region.

2) The dilatation rate dropped to approximately $50 \%$, and the principal axes of contraction have changed their direction from WNW-ESE to N-S around the Yoro fault and to ENE-WSW around the Nobi fault zone.

3) Maximum shear strain rate decreased by about one third around the Yoro fault.

4) Inversion results show that the strain rate change can be well explained by the Tokai slow slip event.

5) The seismicity rate around the Yoro fault dropped to approximately $50 \%$ in the latter half of 2000 .

6) The spatial distribution of $\triangle \mathrm{CFF}$ deduced from our Tokai slow slip model agrees with the reduction in the seismicity rate.

7) Our interpretation is that the seismicity rate which has kept decreasing for several years is mainly controlled by the shear stressing-rate change due to the Tokai slow slip.

Acknowledgments. This study is supported by "Special Project for Earthquake Disaster Mitigation in Urban Areas" by the Ministry of Education, Culture, Sports, Science and Technology, Japan. We thank Dr. Bogdan Enescu, Research Center for Earthquake Prediction, Disaster Prevention Research Institute, Kyoto University for helpful advice. We used GPS data observed by GEONET (GPS Earth Observation Network), which has been operated by the Geographical Survey Institute (GSI), Japan. We used hypocenter data processed by the Japan Meteorological Agency (JMA). We used a program downloaded from U.S.G.S., Earthquake Hazards Program site (http://quake.wr.usgs.gov/research/software/index.html) for the declustering procedure. We used GMT (Generic Mapping Tools) provided by Wessel and Smith (1998) for constructing the figures. We thank two reviewers (S. Miura and T. Parsons) and the editor (T. Sagiya) for their suggestions to improve the manuscript.

\section{References}

Akaike, H., Likelihood and the Bayes procedure, in Bayesian Statistics, edited by J. M. Bernardo, M. H. DeGroot, D. V. Lindley, and A. F. M. Smith, 143-166 pp., University Press, Valencia, Spain, 1980.

Dieterich, J., A constitutive law for rate of earthquake production and its application to earthquake clustering, J. Geophys. Res., 99, 2601-2618, 1994.

Habermann, R. E., Teleseismic detection in the Aleutian Island Arc, $J$. Geophys. Res., 88, 5056-5064,1983.

Harris, R. A. and R. W. Simpson, Changes in static stress on southern California faults after the 1992 Landers earthquake, Nature, 360, 251254, 1992.

Hashimoto, M., Static Stress Changes Associated with the Kobe Earthquake: Calculation of Changes in Coulomb Failure Function and Comparison with Seismicity Change, J. Seismol. Soc. Japan, 48, 521-530, 1995 (in Japanese with English abstract). (Correction to "Static Stress Changes Associated with the Kobe Earthquake: Calculation of Changes in Coulomb Failure Function and Comparison with Seismicity Change", J. Seismol. Soc. Japan, 50, 21-27, 1997.)

Hashimoto, M., K. Onoue, F. Ohya, Y. Hoso, K. Segawa, K. Sato, and Y. Fujita, Crustal deformations in Kii peninsula associated with the SE off the Kii peninsula earthquake sequence of September 5, 2004 derived from dense GPS observations, Earth Planets Space, 57, 1-6, 2005.

Ishida, M., Geometry and relative motion of the Philippine sea plate and Pacific plate beneath the Kanto-Tokai district, Japan, J. Geophys. Res., 97, 489-513, 1992.

Jaume, S. C. and L. R. Sykes, Change in the state of stress on the southern San Andreas fault resulting from the California earthquake sequence of April to June 1992, Science, 258, 1325-1328, 1992.

Kimata, F., K. Hirahara, N. Fujii, and H. Hirose, Repeated occurrence of slow slip events on the subducting plate interface in the Tokai re- 
gion, central Japan, the focal region of the anticipated Tokai earthquake (M=8), EOS Trans. AGU Fall Meeting Suppl., 82, 2001.

Kobayashi, A. and A. Yoshida, Recurrence of the Tokai slow slip inferred from the tide gauge data at Maisaka, J. Geod. Soc. Jpn., 50, 209-212, 2004 (in Japanese with English abstract).

Kobayashi, A., A. Yoshida, T. Yamamoto, and H. Takayama, Slow slip in the focal region of the anticipated Tokai earthquake following the seismo-volcanic event in the northern Izu Islands in 2000, Earth Planets Space, 57, 507-513, 2005.

Matsumura, S., Seismicity rate changes in the inferred locked zone of the Tokai region in the latter half of 1990's, J. Seismol. Soc. Japan, 54, 449463, 2002 (in Japanese with English abstract).

Matsumura, S., Seismicity activity and its change beneath Lake Hamana in the Tokai region (Seismic activity change in the Tokai region: Part 3), J. Seismol. Soc. Japan, 58, 55-65, 2005 (in Japanese with English abstract).

Miyoshi, T. and K. Ishibashi, Geometry of the seismic Philippine Sea slab beneath the region from Ise Bay to western Shikoku, southwest Japan, J. Seismol. Soc. Japan, 57, 139-152, 2004 (in Japanese with English abstract).

Nishimura, T., S. Ozawa, M. Murakami, T. Sagiya, T. Tada, M. Kaidzu, and M. Ukawa, Crustal deformation caused by magma migration in the northern Izu Islands, Japan, Geophys. Res. Lett., 28, 3745-3748, 2001.

Okada, Y., Surface deformation due to shear and tensile faults in a halfspace, Bull. Seismol. Soc. Am., 75, 1135-1154, 1985.

Ozawa, S., M. Murakami, M. Kaidzu, T. Tada, T. Sagiya, Y. Hatanaka, H. Yarai, and T. Nishimura, Detection and monitoring of ongoing aseismic slip in the Tokai region, central Japan, Science, 298, 1009-1012, 2002.

Ozawa, S., S. Miyazaki, T. Nishimura, M. Murakami, M. Kaidzu, T. Imakiire, and X. Ji, Creep, dike intrusion, and magma chamber deflation model for the 2000 Miyake eruption and the Izu islands earthquakes, J. Geophys. Res., 109, doi:10.1029/2003JB002601, 2004.

Ozawa, S., M. Murakami, M. Kaidzu, and Y. Hatanaka, Transient crustal deformation in Tokai region, central Japan, until May 2004, Earth Planets Space, 57, 909-915, 2005.
Reasenberg, P., Second-order moment of Central California seismicity, 1969-1982, J. Geophys. Res., 90, 5479-5495, 1985.

Sagiya, T., S. Miyazaki, and T. Tada, Continuous GPS array and presentday crustal deformation of Japan, Pure. Appl. Geophys., 157, 23032322, 2000.

Shen, Z., D. D. Jackson, and B. X. Ge, Crustal Deformation Across and Beyond the Los Angels Basin from Geodetic Measurements, J. Geophys. Res., 101, 27951-27980, 1996.

Stein, R. S., The role of stress transfer in earthquake occurrence, Nature, 402, 605-609, 1999.

Stein, R. S., G. C. P. King, and J. Lin, Change in failure stress on the southern San Andreas fault system caused by the 1992 magnitude $=7.4$ Landers earthquake, Science, 258, 1328-1332, 1992.

Stein, R. S., G. C. P. King, and J. Lin, Stress triggering of the 1994 M=6.7 Northridge, California earthquake by its predecessors, Science, 265, 1432-1435, 1994.

Toda, S., R. S. Stein, P. A. Reasenberg, J. H. Dieterich, and A. Yoshida, Stress transferred by the $1995 \mathrm{Mw}=6.9$ Kobe, Japan, shock: Effect on aftershocks and future earthquake probabilities, J. Geophys. Res., 103, 24543-24565, 1998.

Toda, S. and R. S. Stein, Response of the San Andreas fault to the 1983 Coalinga-Nuñez earthquake: An application of interactionbased probabilities for Parkfield, J. Geophys. Res., 107, doi: 10.1029/2001JB000172, 2002.

Toda, S., R. S. Stein, and T. Sagiya, Evidence from the AD 2000 Izu islands earthquake swarm that stressing rate governs seismicity, Nature, 419, 58-61, 2002.

Wessel, P. and W. H. Smith, New, improved version of Generic Mapping Tools released, EOS Trans. Am. Geophys. Union, 79, 579, 1998.

Yoshikawa, S., Space and time variation of strain promoted by the slow slip event in the Tokai area revealed by GPS data, Bull. Earthq. Res. Inst., 78, 255-267, 2003.

T. Kobayashi (e-mail: tkoba@mail.sci.hokudai.ac.jp) and M. Hashimoto 